\title{
Le rayonnement de corps noir domestiqué avec lla nanophotonique
}

\author{
Jean-Jacques GREFFET ${ }^{1,2}$, François MARQUIER' \\ 'Laboratoire Charles Fabry, Institut d'Optique, CNRS, Université Paris Sud \\ 2Institut Universitaire de France \\ francois.marquier@institutoptique.fr
}

Le développement de nanostructures permet de répondre au besoin en sources incandescentes infrarouges monochromatiques et directionnelles. Utilisant des matériaux réfléchissants ou des matériaux transparents, ces sources sont de plus modulables rapidement en jouant sur leur température ou leur émissivité. Cet article fait le point sur les technologies actuellement mises en œuvre et présente quelques réalisations.

\section{Vers des sources incandescentes cohérentes}

\section{Les limites des sources incandescentes traditionnelles}

La source de lumière qui nous est la plus familière est le soleil. Il s'agit d'une source de rayonnement qui est remarquablement bien décrite par un rayonnement de corps noir à $5800 \mathrm{~K}$. La source de lumière artificielle la plus répandue est la lampe à filament incandescent qui est portée à des températures comprises entre 1500 et $2800 \mathrm{~K}$ suivant les modèles et les puissances des ampoules. Comme le soleil, ces filaments ont un certain nombre de caractéristiques : la lumière est émise dans un spectre très large qui ne dépend que de la température, la lumière émise est non polarisée et est émise dans toutes les directions. Pour un grand nombre d'applications, ces propriétés peuvent présenter des inconvénients. Par exemple, pour de l'éclairage, on ne souhaite émettre que dans la partie visible du spectre. Or, une très faible partie du spectre émis par une lampe à incandescence se trouve dans le spectre visible de sorte qu'une partie de l'énergie émise est inutile. C'est l'un des facteurs qui explique que le rendement typique en terme de puissance lumineuse utile normalisée par la puissance électrique consommée ne soit que de quelques pour cents. Ces faibles performances ont conduit l'Union européenne à programmer le retrait progressif des lampes incandescentes du marché de l'éclairage. Il existe aujourd'hui des solutions alternatives utilisant des luminophores ou des diodes électroluminescentes dont le rendement est très supérieur à celui des ampoules incandescentes.

\section{Des sources infrarouge indispensables pour de nombreuses applications}

Cependant, ces solutions ne sont pas utilisables dans l'infrarouge (IR). Par exemple, le rendement d'une diode chute d'environ trois ordres de grandeur lorsque l'on passe d'une longueur d'onde de $500 \mathrm{~nm}$ à une longueur d'onde de $5 \mu \mathrm{m}$. Ceci est lié à une propriété fondamentale: le taux d'émission spontanée varie comme le cube de la fréquence. C'est pourquoi, mis à part les lasers à cascade quantique et les oscillateurs paramétriques qui sont des objets de laboratoire, les sources IR disponibles actuellement sont encore des filaments incandescents. Ces sources sont couramment utilisées pour réaliser de la spectroscopie de transmission pour caractériser des gaz. On a également besoin de sources de ce type pour le séchage ou pour le chauffage radiatif. Enfin, des sources infrarouges monochromatiques sont utiles dans le contexte de la production d'énergie thermo-photovoltaïque. II s'agit de convertir de la chaleur (issue d'une installation industrielle, d'un brûleur ou d'un concentrateur solaire) en rayonnement monochromatique de fréquence $v$ pour éclairer une cellule photovoltaïque dont la bande interdite est $\mathrm{E}_{\mathrm{g}}=\mathrm{hv}$. L'accord entre la fréquence d'émission et la largeur de la bande interdite permet d'envisager un rendement élevé.

\section{Une solution : des sources incandescentes partiellement cohérentes}

L'objet de cet article est de montrer qu'en fabriquant des nanostructures judicieusement choisies, il devient possible de créer de véritables métamatériaux qui sont capables d'émettre du rayonnement de corps noir uniquement aux fréquences souhaitées et dans les directions souhaitées. Ceci peut paraître surprenant au premier abord, la directivité et le caractère monochromatique étant le plus souvent l'apanage des sources laser. Pourtant, il est possible de réaliser des sources incandescentes partiellement cohérentes : elles peuvent être directives, à spectre étroit et modulables à très haute fréquence comme nous allons le voir. Cependant, le nombre de photons par mode est donné par la distribution de Bose-Einstein. II s'ensuit que les propriétés statistiques de la lumière sont celles du corps noir et non 
pas d'un laser. De même, la luminance est toujours très inférieure à celle d'un laser. En d'autres termes, le nombre moyen de photons par mode est très inférieur à un, ce qui est très différent d'un laser.

\section{Comment modéliser l'émission de lumière}

Afin de comprendre comment faire pour concevoir des sources de rayonnement IR, il est nécessaire de disposer d'un modèle de l'émission thermique. Pour les besoins de cet article, nous allons nous contenter de faire appel à la radiométrie. Dans ce cadre, le rayonnement émis par une source s'exprime comme le produit de deux facteurs. Le premier ne dépend que de la température et exprime la puissance émise par unité de surface et d'angle solide. C'est ce que l'on appelle la luminance de corps noir qui est notée $L^{\circ}(\lambda, T)$. Le second terme caractérise l'émetteur et est appelé émissivité. C'est un nombre sans dimension noté $\varepsilon$. C'est une fonction réelle positive qui dépend de la longueur d'onde, de l'angle et de la polarisation. On peut montrer que l'émissivité est égale à l'absorptivité. Cette relation appelée loi de Kirchhoff impose que l'émissivité soit inférieure à 1. Elle exprime un lien entre la capacité à émettre thermiquement du rayonnement et la capacité à l'absorber. II en résulte que la conception d'un émetteur sélectif estéquivalente à la conception d'un absorbeur sélectif.

\section{Concevoir une source monochromatique}

\section{L'apport de la nanophotonique}

Voyons comment utiliser cette relation pour imaginer la conception d'une source à spectre étroit. L'idée de base est de partir d'un matériau qui n'est pas absorbant. Ce peut être un miroir métallique réfléchissant sur tout le spectre IR ou bien un matériau semi-conducteur transparent dans tout le spectre IR. A partir de là, l'idée consiste à introduire une structure qui absorbe à une seule fréquence IR et faire en sorte que l'absorption soit quasi totale. En vertu de la loi de Kirchhoff, il suffit alors de chauffer cette structure pour qu'elle émette uniquement à la bonne fréquence. Comment parvenir à réaliser cela ? C'est à ce stade que la nanophotonique s'avère utile.

\section{Utilisation d'un matériau réfléchissant}

Un premier exemple de réalisation est représenté à la figure 1a. On y voit des parallélépipèdes de longueur et largeur micrométriques, d'épaisseur submicronique. Ces structures se comportent comme des résonateurs pour le champ électromagnétique. En modifiant leurs longueurs, il est possible de contrôler la fréquence de résonance de la même façon que l'on change la note d'un tuyau d'orgue en changeant son diamètre et sa longueur. Ainsi, il est possible d'optimiser les paramètres géométriques par

\section{HORI8A Imagerie multimodale Scientific Intégration d'AFM et spectroscopie}

$\mathrm{L}$

e microscope à force atomique (AFM) permet l'investigation de multiples propriétés physiques de la surface des matériaux, mais n'est pas capable de déterminer la composition chimique moléculaire correspondante, tandis que les méthodes spectroscopiques telles de la spectroscopie Raman permettent cette identification.

L'intégration de ces techniques en une plateforme d'imagerie multimodale permet l'investigation de matériaux nanoscopiques avec une grande variété de méthodes.

Les principales méthodes incluent :

- AFM (contact, non-contact)

- STM

- Shear-Force

- Spectroscopie Raman, de fluorescence ou photoluminescence

- Mesures co-localisées et mesures en champ proche (NSOM, TERS)

\section{Applications :}

- Caractérisation de polymères

- Analyse de surface de nanostructures (photoniques, semi-conducteurs...)

- Biologie, imagerie cellulaire

- Capteurs photoniques ou utilisant des effets plasmoniques (SERS)

- Nanomatériaux en général (graphene, nanotubes de carbone, nano fils, nano rubans etc.)

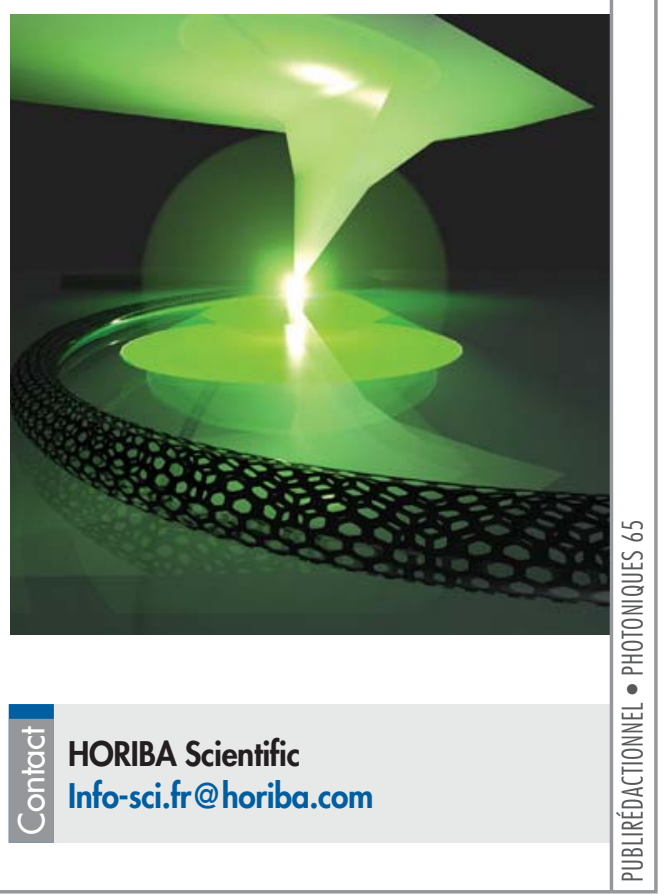



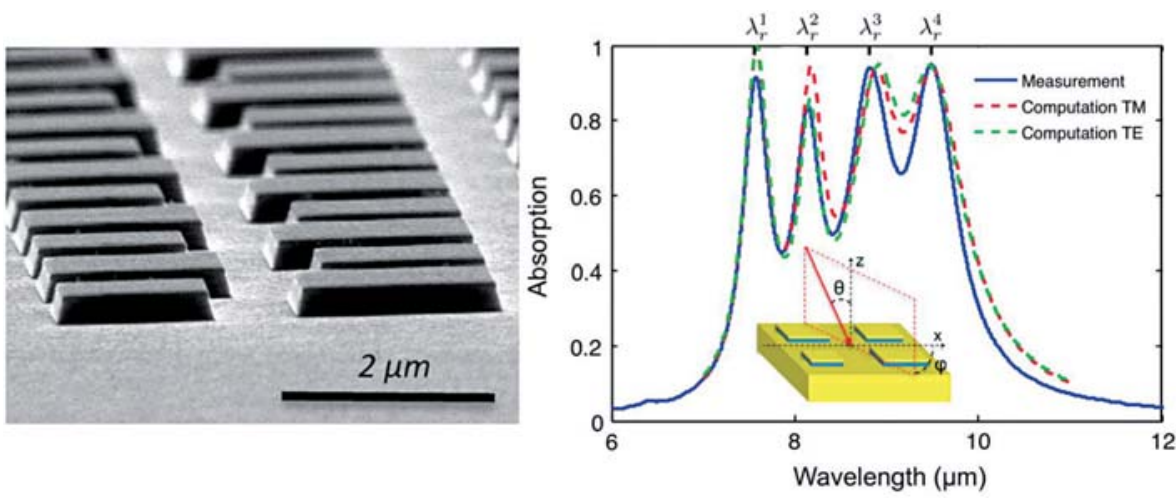

Figure 1. Métamatériau pour absorbant/émetteur sélectif en fréquence. L'insert de la figure de droite montre les structures de ZnS (en bleu) déposées sur un substrat d'or et recouvertes d'un film de $50 \mathrm{~nm}$ d'or. Les tailles latérales sont de l'ordre du micomètre comme le montre le cliché obtenu en microscopie électronique à balayage de gauche. Le spectre d'absorption présente des pics qui peuvent être associés aux résonances des objets individuels (d'après [1]).

modélisation numérique de sorte que l'absorption soit quasi monochromatique à une longueur d'onde $\lambda$. Il est également possible d'obtenir une surface ayant une absorption totale à plusieurs fréquences. Pour cela, on utilise une propriété remarquable des structures résonantes. Pour caractériser leur absorption, on définit une « aire effective » appelée section efficace d'absorption et notée $\sigma$ telle que la puissance incidente par unité de surface multipliée par $\sigma$ soit égale à la puissance absorbée. À résonance, la section efficace est de l'ordre de $\lambda^{2}$ même si la taille réelle est beaucoup plus petite. Ainsi, il suffit de placer des absorbeurs résonnants espacés de $\lambda$ pour obtenir une absorption totale. II est alors possible de placer sur une surface de l'échantillon de l'ordre de $\lambda^{2}$, plusieurs objets résonants à différentes fréquences tout en conservant une absorption quasi totale à chaque longueur d'onde comme on le voit à la figure $1 b$.

\section{Utilisation d'un matériau transparent}

Une deuxième façon de concevoir une source monochromatique à la longueur d'onde I peut être nevisagée à partir non pas d'un miroir mais d'un milieu transparent dans I'IR tel qu'un semi-conducteur. Pour introduire de l'absorption, nous faisons là encore appel à la nanophotonique. Il est possible d'implanter des puits quantiques tels que par exemple des couches de quelques nanomètres d'épaisseur de GaAs séparées par des barrières de AlGaAs. Les électrons confinés dans ces couches appelées puits quantiques ont des niveaux d'énergie tels que des transitions peuvent apparaître à la fréquence voulue. À ce stade, un matériau qui comporte une absorption intrinsèque à une fréquence donnée a été créé. Rien ne garantit que la lumière éclairant l'interface air/semi-conducteur ne sera pas réfléchie. Pour éviter cela, il est possible de graver un réseau de trous créant ainsi ce que I'on appelle un cristal photonique. Cela a deux effets : le premier est de modifier la propagation des ondes dans le milieu et d'y créer des résonances qui favorisent l'absorption si les puits quantiques sont accordés en fréquence au cristal photonique; le second est de modifier le couplage au milieu extérieur. La figure $2 b$ montre que cette approche a permis de réaliser des sources à spectre très étroit et qui émettent très peu en dehors de la zone visée.
Dans les deux cas présentés, la structuration de la surface est périodique de sorte que ces structures apparaissent comme des réseaux. Cependant, la période est inférieure à la demi longueur d'onde d'émission $\lambda / 2$ de sorte que seul l'ordre zéro existe. Ces surfaces se comportent donc comme des interfaces de matériaux effectifs ayant des propriétés qui ont été conçues à volonté : on parle de métamatériaux.

\section{Des sources incandescentes directionnelles}

\section{Le phénomène d'absorption directionnelle}

S'il est possible de fabriquer des sources incandescentes monochromatiques, on peut aussi se poser la question de la direction d'émission. Le contrôle de ce paramètre permet de gagner encore sur l'efficacité globale de la source. L'idée est que le rayonnement émis à une longueur d'onde donnée puisse être envoyé dans la direction utile et seulement dans cette direction. Pour comprendre comment cela est possible, nous pouvons encore nous appuyer sur la loi de Kirchhoff. II existe des réseaux métalliques pour lesquels on observe une absorption totale pour un angle d'incidence particulier et une fréquence particulière. Cette absorption résonnante apparaît lorsque l'onde incidente se couple à une onde de surface appelée plasmon de surface. La sélectivité angulaire résulte de ce que le couplage ne peut se faire que pour un angle d'incidence bien précis, donné par

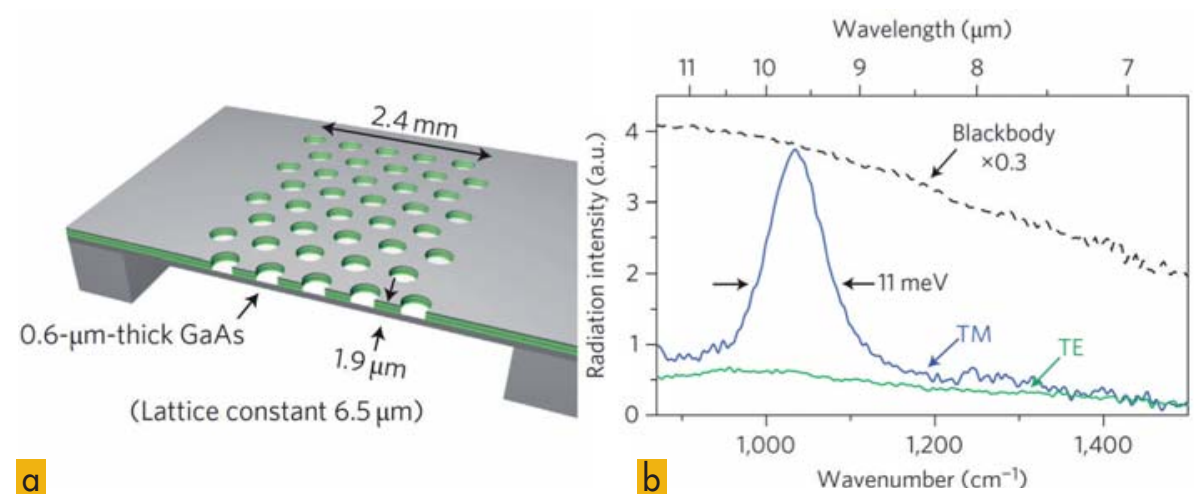

Figure 2. Émetteur sélectif en fréquence. (a) Vue de la membrane absorbante : un ensemble de 63 puits quantiques de GaAs dans des barrières de AlGaAs est déposé sur un substrat de GaAs. Un cristal photonique est ensuite gravé. (b) Spectre d'émission. Un pic étroit d'émissivité de 0,3 est obtenu (d'après [2]). 
la loi des réseaux. Du fait de la présence de mécanismes de perte dans les métaux, I'onde de surface est absorbée, c'est-à-dire transformée en chaleur. Dans le cas des métaux, l'onde de surface est associée à une oscillation collective des électrons. Dans le cas des diélectriques, il existe également des ondes de surface associées à des oscillations collectives des atomes : les phonons polaritons de surface.

\section{Réalisation d'émetteurs directionnels}

D’après l'égalité entre émissivité et absorptivité énoncée par la loi de Kirchhoff, il est possible de tirer parti de cette absorption directionnelle pour concevoir des émetteurs directionnels. La première démonstration de cette émission directionnelle a été réalisée avec du SiC dans l'infrarouge moyen vers $10 \mu \mathrm{m}$ [3]. Depuis, de nombreuses autres réalisations ont été décrites. La figure 3 montre deux exemples de réseaux de tungstène, l'un lamellaire, I'autre cylindrique qui forment des sources thermiques directionnelles aux longueurs d'onde indiquées. Ces exemples sont pris parmi de nombreuses réalisations qui émergent depuis les

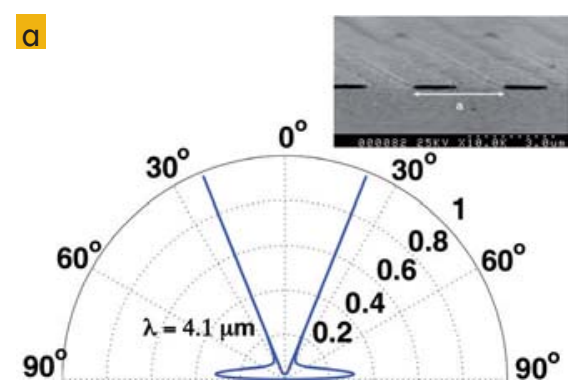

\section{OSICS T100 Lasers Accordables Compacts de 1260 à 1680 nm}

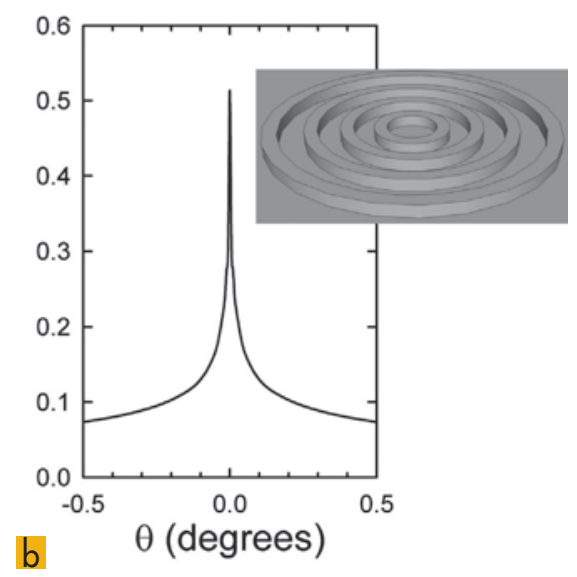

Figure 3. Émissivité en fonction de l'angle d'observation pour deux réseaux de tungstène. (a) un réseau lamellaire de période 3 microns (d'après [4]) (b) un réseau cylindrique de période 3,5 microns (d'après [5]).

- $1100 / 1550: 1490-1610 \mathrm{~nm}$

\section{Le rayonnement thermique peut-il être cohérent?}

Nous venons de voir que les sources incandescentes peuvent émettre dans une gamme de fréquences limitée et dans une gamme de directions limitée. Ceci revient à dire que ce sont des sources qui sont partiellement cohérentes temporellement et spatialement. Or, les sources incandescentes sont volontiers citées lorsque l'on cherche un exemple de source incohérente. Cette apparente contradiction mérite que l'on s'y arrête.

Pourquoi dit-on qu'une source thermique est incohérente ? La raison est liée au fait que les sources microscopiques du rayonnement à l'équilibre thermodynamique sont des processus d'émission spontanée. Ces processus se font localement à l'échelle de chaque électron ou atome.

La cohérence observée résulte de ce que lors de la conception des sources, nous avons inclus des filtres fréquentiels (cohérence temporelle) et des filtres sélectionnant de directions d'émission (cohérence spatiale). On peut alors se poser la question de l'origine microscopique de cette cohérence. Dans le cas temporel, la cohérence traduit l'existence d'un temps de cohérence dans la source associé à un mode collectif (plasmon dans le cas des électrons, phonon dans le cas des mouvements des noyaux). À l'évidence, ce temps de cohérence provient de l'introduction dans la source de résonateurs qui ont un facteur de qualité et donc une durée de vie qui y est associée. C'est cette rémanence de l'excitation dans le résonateur qui construit la cohérence temporelle. Quand bien même le rayonnement thermique a un bruit à spectre très large donné par la distribution de Bose-Einstein, les courants induits dans le matériau ont un spectre plus étroit car il est filtré par la résonance des structures.

Dans le cas spatial, la directivité traduit l'existence d'une cohérence du champ le long de la surface de l'émetteur. Ici, l'existence de corrélations spatiales du champ sur des distances de plusieurs longueurs d'onde résulte de ce qu'une source ponctuelle peut exciter des ondes de surface qui décroissent exponentiellement avec une longueur caractéristique $I$. Les champs de ces ondes de surface polarisent la matière et induisent ainsi des courants le long de la surface créant une corrélation du champ sur une échelle de longueur / également. On peut alors montrer que la directivité varie comme $\lambda / I$.

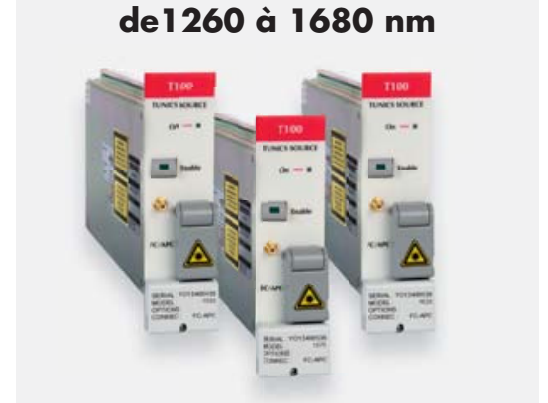

6 modèles OSICS T100 :

- $\mathrm{T} 100 / 1310: 1260-1360 \mathrm{~nm}$

- $1100 / 1415$ : 1360 - 1470 nm

- T100/1520 : 1465 - 1575 nm

- $\mathrm{T} 100 / 1575$ : 1520 - $1630 \mathrm{~nm}$

- $\mathrm{T} 100 / 1620$ : 1560 - $1680 \mathrm{~nm}$

Caractéristiques OSICS T100:

- Puissance optique $>3 \mathrm{dBm}$

- Dynamique laser/SSE : $90 \mathrm{~dB}$

- Précision en $\lambda<0,2 \mathrm{~nm}$

- Mode pas à pas

- Prix attractif

\section{OSICS Full-Band Laser}

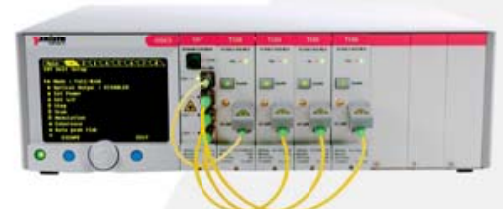

Le laser couvrant toute la bande télécom

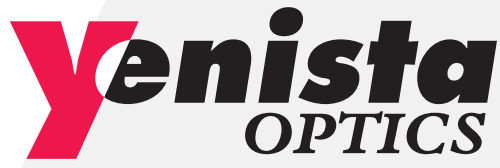

Tél. : +33 (0)296483716 sales-emea@yenista.com www.yenista.com 
années 2000 avec différents matériaux/ structures/résonances.

\section{Des sources incandescentes modulables rapidement}

\section{Modulation de la température}

Un facteur de mérite important d'une source de lumière est sa capacité à être modulée rapidement. Pour une source incandescente, la première idée est de jouer sur la modulation de la température pour réaliser cette fonction. Reprenons l'exemple des ampoules à incandescence de notre vie quotidienne. Nous savons que le courant alternatif délivré en France oscille à une fréquence de $50 \mathrm{~Hz}$. Les filaments chauffant grâce à l'effet Joule, la température du filament, et donc le rayonnement IR émis par ces sources, a une intensité qui oscille à $100 \mathrm{~Hz}$, comme le carré du courant. II est difficile de monter vraiment plus haut en fréquence dans le rayonnement thermique. Les phénomènes de relaxation de la température ont en effet des constantes de temps de I'ordre de quelques millisecondes. En modulant plus vite le courant, la température n'aurait pas le temps de diminuer et on observerait simplement un rayonnement constant.

\section{Modulation de l'émissivité}

Une deuxième idée est d'agir sur l'émissivité du matériau plutôt que sur sa température. Le schéma de fonctionnement est le suivant: on garde le matériau à une température donnée, suffisante pour avoir un rayonnement thermique utile, et on module électriquement l'amplitude de l'émissivité qui, jouant le rôle d'un interrupteur, coupe ou laisse passer le rayonnement thermique. Une première démonstration astucieuse de ce concept a été publiée récemment [6,7]. La source considérée est constituée d'un puits quantique unique de GaAs. Dans le lointain infrarouge, ce film de GaAs a des modes électromagnétiques à ses interfaces. De plus, autour d'une longueur d'onde de 34 microns, le GaAs a une fonction diélectrique e très proche de zéro. Cette propriété peut conduire à exalter fortement

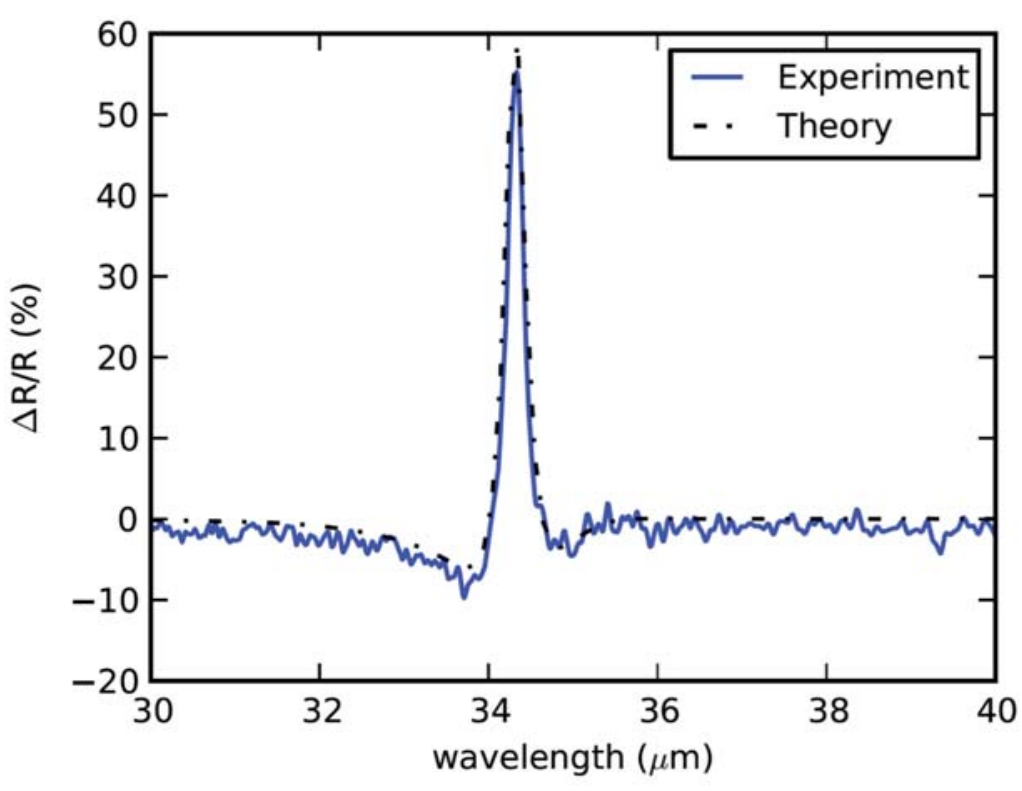

Figure 4. Variation relative de la réflectivité d'une structure optimisée autour d'un puits quantique de GaAs lorsque l'on fait varier une tension de commande appliquée à la structure. La loi de Kirchhoff nous dit que l'émissivité présente aussi une variation forte à la longueur d'onde du pic (d'après [6]).

le champ électrique confiné dans le GaAs, on parle d'effet ENZ, pour Epsilon Near Zero. Cette propriété résulte du fait que le produit eEn doit rester constant du fait de la continuité des champs normaux aux interfaces. Ce mode confiné, excité thermiquement, peut être couplé à des ondes propagatives à l'aide d'un réseau : on extrait alors de l'énergie par rayonnement de la structure. En appliquant une tension entre deux électrodes de chaque côté du puits, il est possible de changer la population d'électrons dans le puits et donc la constante diélectrique : l'effet d'exaltation n'existe plus et le mode confiné disparaît quasiment. La structure ne rayonne plus. La figure 4 montre une preuve de concept obtenue récemment. L'amplitude de modulation est assez faible avec ce dispositif. Cependant, on voit que l'on dispose d'un dispositif fonctionnant à température ambiante dont la fréquence de modulation n'est limitée que par la vitesse avec laquelle on peut modifier la densité de porteurs dans un puits quantique. Ceci est un problème analogue à la technologie des transistors qui fonctionnent à des cadences approchant le GHz. Des fréquences de l'ordre du $\mathrm{MHz}$ ou $\mathrm{GHz}$ sont envisageables, même si elles restent à être montrées expérimentalement.

\section{Références}

[1] P. Bouchon, C. Koechlin, F. Pardo, R. Haidar and J.L. Pelouard, Wideband omnidirectional infrared absorber with a patchwork of plasmonic nanoantennas, Optics Letters, 37, 1038 (2012)

[2] M. De Zoysa, T. Asano, K. Mochizuki, A. Oskooi, T. Inove and S. Noda, Conversion of broadband to narrowband thermal emission through energy recycling, Nature Photonics, 6 , 535 (2012)

[3] J.J. Greffet, R. Carminati, K. Joulain, J.P. Mulet, S. Mainguy and Y. Chen, Coherent emission of light by thermal sources, Nature, 416, 61 (2002)

[4] S.E. Han and D.J. Norris, Beaming thermal emission from hot metallic bull's eyes, Optics Express, 18, 4829 (2010)

[5] M. Laroche, C. Arnold, F. Marquier, R. Carminati, J.J. Greffet, S. Collin, N. Bardou, and J.L. Pelouard, Highly directional radiation generated by a tungsten thermal source, Optics Letters, 30, 2623 (2005)

[6] S. Vassant, A. Archambault, F. Marquier, F. Pardo, U. Gennser, A. Cavanna, J.L. Pelouard, and J.J. Greffet, Epsilon-Near-Zero Mode for Active Optoelectronic Devices, Physical review Letters, 109, 237401 (2012)

[7] S. Vassant, I. Moldovan Doyen, F. Marquier, F. Pardo, U. Gennser, A. Cavanna, J.L. Pelouard, and J. J. Greffet, Electrical modulation of emissivity, Applied Physics Letters, 102, 081125 (2013) 